\title{
A New Fractional Calculus Model for the Two-dimensional Anomalous Diffusion and its Analysis
}

\author{
Yu. Luchko * \\ Department of Mathematics, Physics, and Chemistry \\ Beuth Technical University of Applied Sciences Berlin \\ Luxemburger Str. 10, 13353 Berlin, Germany
}

\begin{abstract}
In this paper, a special model for the two-dimensional anomalous diffusion is first deduced from the basic continuous time random walk equations in terms of a time- and spacefractional partial differential equation with the Caputo time-fractional derivative of order $\alpha / 2$ and the Riesz space-fractional derivative of order $\alpha$. For $\alpha<2$, this $\alpha$-fractional diffusion equation describes the so called Lévy flights that correspond to the continuous time random walk model, where both the mean waiting time and the jump length variance of the diffusing particles are divergent. The fundamental solution to the $\alpha$-fractional diffusion equation is shown to be a two-dimensional probability density function that can be expressed in explicit form in terms of the Mittag-Leffler function depending on the auxiliary variable $|x| /(2 \sqrt{t})$ as in the case of the fundamental solution to the classical isotropic diffusion equation. Moreover, we show that the entropy production rate associated with the anomalous diffusion process described by the $\alpha$-fractional diffusion equation is exactly the same as in the case of the classical isotropic diffusion equation. Thus the $\alpha$-fractional diffusion equation can be considered to be a natural generalization of the classical isotropic diffusion equation that exhibits some characteristics of both anomalous and classical diffusion.
\end{abstract}

Keywords and phrases: anomalous diffusion, continuous time random walks, fractional diffusion equation, Mellin-Barnes integral, Mittag-Leffler function, two-dimensional probability density function, entropy, entropy production rate

Mathematics Subject Classification: 26A33, 35C05, 35E05, 35L05, 45K05, 60E99

\section{Introduction}

The so-called non-Brownian or anomalous diffusion is nowadays an important topic both in mathematics and in many different applications (see e.g. the recent survey paper [23] for about three hundred references to the relevant works). Usually, the anomalous diffusion processes are defined as those that do not longer follow the Gaussian statistics on the long time intervals. Especially, the linear time dependence of the

\footnotetext{
${ }^{*}$ Corresponding author. E-mail: luchko@beuth-hochschule.de
} 
mean squared displacement of the diffusing particles does not hold any more and has to be either replaced with a different (mainly power-law) dependence or the mean squared displacement does not exist at all.

The probably most considerable models of the anomalous diffusion processes are the continuous time random walks on the micro-level and different types of fractional differential equations that are obtained from the underlying stochastic models for some special jump probability density functions with infinite first or/and second moments on the macro-level (see e.g. [10] [17], [24]). In this connection, the so called time-fractional diffusion equation that is obtained from the classical diffusion equation by replacing the first order time derivative by a fractional derivative of order $\alpha, 0<\alpha<1$ has to be especially mentioned. In [32], the Green function for the time-fractional diffusion equation was shown to be a probability density function evolving in time with the mean squared displacement of the diffusing particles of the form $C_{\alpha} t^{\alpha}, 0<\alpha<1$. Thus the time-fractional diffusion equation can be considered to be a suitable mathematical model for the sub-diffusion processes. Moreover, the entropy production rate of the anomalous diffusion processes that are described by the time-fractional diffusion equation was shown to be linearly depending on the derivative order $\alpha$ and thus growing with increasing of $\alpha$ from zero (timeindependent processes) to one (diffusion processes) ([8], [11]). From the viewpoint of the continuous time random walks, the time-fractional diffusion equation corresponds to the case of the long-tailed waiting time probability density function with the long time asymptotics of the form $O\left(t^{-1-\alpha}\right), t \rightarrow \infty$. This means in particular that for $0<\alpha<1$ the mean waiting time does not exists for this long-tailed waiting time probability density function ([24], [25]).

In the literature, many other kinds of time-, space, or time and space-fractional differential equations were derived from the CTRW models and suggested as models for anomalous diffusion processes (see e.g. [3], [20], [26], [27], [28], [33] and the references therein). In particular, the case of the waiting time probability density function and the jump length probability density function with the same powerlaw asymptotic behavior has been considered in [16]. Under some standard assumptions, the so called fractional wave equation can be asymptotically derived from the continuous time random walk model mentioned above (see [16] for details). The solutions of the fractional wave equation (see e.g. [2]) can be interpreted as the damped waves with the constant propagation velocities that means that this equation inherits some characteristics of the wave equation, too ([13]-[14]). Otherwise, the fundamental solution of the one-dimensional fractional wave equation is known to be a spatial probability density function evolving in time and thus is related to the diffusion processes [15].

In this paper, we consider another special case of the waiting time probability density function and the jump length probability density function in the framework of the continuous time random walk model that leads to the $\alpha$-fractional diffusion equation on the macro-level. This equation contains the Caputo time-fractional derivative of order $\alpha / 2$ and the Riesz space-fractional derivative of order $\alpha$ and models a special case of the anomalous diffusion. From the viewpoint of the continuous time random walks, the $\alpha$-fractional diffusion equation describes the Lévy flights, where both the mean waiting time and the jump length variance of the diffusing particles are divergent. In this paper, we focus on the twodimensional $\alpha$-fractional diffusion equation that possesses some remarkable properties that do not appear in the one- and three-dimensional cases and discuss some properties of its fundamental solution that were not yet mentioned in the literature. It turns out that the fundamental solution to this equation can be interpreted as a two-dimensional probability density function evolving in time. Moreover, as in the case of the fundamental solution to the classical isotropic diffusion equation, the fundamental equation to the $\alpha$ fractional diffusion equation depends on the auxiliary variable $|x| /(2 \sqrt{t})$ and can be expressed in explicit form in terms of the Mittag-Leffler function. Even if the $\alpha$-fractional diffusion equation is associated with the anomalous diffusion processes, the entropy production rate of its fundamental solution is the same as in the case of the classical isotropic diffusion equation. Thus the $\alpha$-fractional diffusion equation can be considered to be a natural generalization of the classical isotropic diffusion equation that exhibits some characteristics of both anomalous and conventional diffusion. Let us mention that the $\alpha$-fractional diffusion equation is a particular case of the more general time- and space-fractional diffusion equation that has been analyzed in the one-dimensional case in [21] and in the multi-dimensional case in [6] and [29] 
to mention only few of many relevant publications. In [12], the case of the one-dimensional $\alpha$-fractional diffusion equation was considered in detail.

The rest of the paper is organized as follows. In the second section, basic definitions, problem formulation, and some analytical results for the initial-value problems for the two-dimensional $\alpha$-fractional diffusion equation are presented. In particular, some integral representations for the fundamental solution $G_{\alpha}$ of the two-dimensional $\alpha$-fractional diffusion equation are derived. The Mellin-Barnes integral representation of $G_{\alpha}$ is then employed to express it in terms of the Mittag-Leffler function for all values of $\alpha, 0<\alpha \leq 2$. The third section is devoted to interpretation of the fundamental solution $G_{\alpha}$ as a probability density function evolving in time for all values of $\alpha, 0<\alpha \leq 2$. Moreover, it is shown that the entropy production rate of the stochastic process governed by the $\alpha$-fractional diffusion equation is the same as for the classical two-dimensional diffusion equation. To illustrate the analytical findings, some results of numerical calculations, numerous plots, their physical interpretation and discussion are presented in the last section.

\section{Continuous time random walk model}

In this section, we shortly remind the reader of the general framework of the continuous time random walk model and deduce from this model the $\alpha$-fractional diffusion equation under some suitable assumptions posed on the waiting time probability density function and the jump length probability density function. To make the presentation shorter, we restrict ourselves to the one-dimensional case. The $n$-dimensional case can be considered following the lines of the one-dimensional case.

Let us consider a one-dimensional random walker (diffusing particle) whose initial location at the time $t=0$ is described by the probability density function $u_{0}=u_{0}(x)$ and who then randomly jumps at the time instants $t=t_{n}, n=1,2, \ldots$ either to the left or to the right, thereby covering the distance $\Delta x_{n}, n=1,2, \ldots$. We are interested in describing the stochastic process associated with the movement of the random walker and in particular in determination of the probability density function $u=u(x, t)$ of being at position $x$ at time $t$.

The main idea behind the continuous time random walk model is that the length of a given jump, as well as the waiting time elapsing between two successive jumps are governed by a joint probability density function (pdf) $\psi=\psi(x, t)$ which will be referred to as the jump pdf. From the jump pdf $\psi$, the jump length pdf

$$
\lambda(x)=\int_{0}^{\infty} \psi(x, t) d t
$$

and the waiting time pdf

$$
w(t)=\int_{-\infty}^{\infty} \psi(x, t) d x
$$

can be determined.

In what follows we deal with the uncoupled continuous time random walks with the jump pdfs in the form $\psi(x, t)=w(t) \cdot \lambda(x)$.

The two important characteristics of the continuous time random walk processes are the mean waiting time

$$
T=\int_{0}^{\infty} w(t) t d t
$$

and the jump length variance

$$
\Sigma^{2}=\int_{-\infty}^{\infty} \lambda(x) x^{2} d x
$$

They can be finite or infinite and this makes the difference between the different continuous time random walk processes.

It is well-known that the probability density function $u=u(x, t)$ of being at position $x$ at time $t$ can be described by the master equations of the convolution type (see e.g. [9], [25]). To write down these 
equations, let us denote by $\eta=\eta(x, t)$ the pdf of the event that the random walker just arrived at position $x$ at time $t$. Then $\eta$ satisfies the equation (see e.g. [9], [25])

$$
\eta(x, t)=\int_{-\infty}^{+\infty} \int_{0}^{t} \eta\left(x^{\prime}, t^{\prime}\right) \lambda\left(x-x^{\prime}\right) w\left(t-t^{\prime}\right) d t^{\prime} d x^{\prime}+u_{0}(x) \delta(t) .
$$

The pdf $u=u(x, t)$ of being at position $x$ at time $t$ is then given by the formula

$$
u(x, t)=\int_{0}^{t} \eta\left(x, t^{\prime}\right) \Psi\left(t-t^{\prime}\right) d t^{\prime}
$$

where

$$
\Psi(t)=1-\int_{0}^{t} w\left(t^{\prime}\right) d t^{\prime}
$$

is assigned to the probability of no jump event during the time interval $(0, t)$.

The integral equations (2.5)-(2.7) determine the one-point probability density function that is an important part of a mathematical model but of course not enough to fully characterize the underlaying stochastic process (see e.g. [1] for more details) in contrast to the situation with the generalized grey Brownian motion that is fully characterized by the master equations ([28]). To determine the probability density function $u=u(x, t)$ let us first apply the Fourier and the Laplace transforms to these equations. By using the well-known product properties of the Fourier and the Laplace transforms when applying them to the Fourier or Laplace convolutions respectively, we can deduce from the equations (2.5)-(2.7) the Fourier-Laplace transform of the pdf $u=u(x, t)$ :

$$
\widehat{\widetilde{u}}(\kappa, s)=\frac{1-\widetilde{w}(s)}{s} \frac{\widehat{u_{0}}(\kappa)}{1-\widehat{w}(s) \widetilde{\lambda}(\kappa)},
$$

where $\widehat{u_{0}}(\kappa)$ denotes the Fourier transform of the initial condition $u_{0}(x)=u(x, 0)$. It is worth to mention that a purely probabilistic proof of this equation is given in [1].

We remind the reader that the Fourier transform of a function $f: \mathbb{R}^{n} \rightarrow \mathbb{R}$ is defined by

$$
\widehat{f}(\kappa)=\mathcal{F}\{f(x) ; \kappa\}=\int_{\mathbb{R}^{n}} e^{+i \kappa \cdot x} f(x) d x, \kappa \in \mathbb{R}^{n},
$$

and the Laplace transform of a function $f: \mathbb{R}_{+} \rightarrow \mathbb{R}$ by

$$
\widetilde{f}(s)=\mathcal{L}\{f(t) ; s\}=\int_{0}^{\infty} e^{-s t} f(t) d t, \quad s \in \mathbb{C} .
$$

It can be shown (see e.g. [25]) that if both the mean waiting time and the jump length variance are finite, then the long-time limit behavior of this continuous time random walk corresponds to the Brownian motion.

Now let us discuss a specific continuous time random walk, where both the mean waiting time and the jump length variance do not exist. Moreover, we suppose that the asymptotic behavior of the waiting time and the jump length pdfs has following form $(1<\alpha<2)$ :

$$
\begin{gathered}
w(t) \approx A_{\alpha}(t / \tau)^{-1-\frac{\alpha}{2}}, t \rightarrow+\infty, \quad t / \tau \gg 1, \\
\lambda(x) \approx B_{\alpha}(|x| / \sigma)^{-1-\alpha}, \quad|x| \rightarrow \infty, \quad|x| / \sigma \gg 1 .
\end{gathered}
$$

The Tauberian theorems for the Laplace and the Fourier transforms lead to the asymptotic formulas for the transformed pdfs:

$$
\widetilde{w}(s) \approx 1-(\tau s)^{\frac{\alpha}{2}}, s \rightarrow 0,
$$




$$
\widehat{\lambda}(\kappa) \approx 1-(\sigma|\kappa|)^{\alpha}, \kappa \rightarrow 0 .
$$

It is important to mention that the specific form of both pdfs is of minor importance. In particular, the waiting time pdf can be taken without loss of generality in the form of the Mittag-Leffler survival probability (see [7])

$$
w(t)=-\frac{d}{d t} E_{\frac{\alpha}{2}}\left(-t^{\frac{\alpha}{2}}\right), \quad E_{\beta}(x)=\sum_{k=0}^{\infty} \frac{x^{k}}{\Gamma(\beta k+1)}, \beta>0 .
$$

The Laplace transform of the Mittag-Leffler survival probability is known to be

$$
\widetilde{w}(s)=\frac{1}{1+s^{\frac{\alpha}{2}}}
$$

and thus has the desired asymptotic behavior.

As to the jump length pdf, one of the possible candidates would be the Lévy pdf with the Fourier transform

$$
\widehat{\lambda}(\kappa)=\exp \left(-\sigma^{\alpha}|\kappa|^{\alpha}\right) \approx 1-\sigma^{\alpha}|\kappa|^{\alpha}, 1<\alpha<2,|\kappa| \rightarrow 0 .
$$

Substituting the asymptotical formulas for the transformed waiting time and jump length pdfs into the formula (2.8) we get the asymptotics of the Fourier-Laplace transform of the pdf $u=u(x, t)$ in the form

$$
\widehat{\widetilde{u}}(\kappa, s) \approx \frac{\widehat{u_{0}}(\kappa) / s}{1+C_{\alpha} s^{-\frac{\alpha}{2}} \kappa^{\alpha}}, s \rightarrow 0, \kappa \rightarrow 0 .
$$

Using the Tauberian theorems for the Laplace and Fourier transforms, the last equation can be transformed to a time- and space-fractional partial differential equation for $t / \tau \gg 1$ and $|x| / \sigma \gg 1$.

Indeed, after multiplication with the denominator of the right-hand side, the equation (2.13) becomes

$$
\left(1+C_{\alpha} s^{-\frac{\alpha}{2}} \kappa^{\alpha}\right) \widehat{\widetilde{u}}(\kappa, s) \approx \widehat{u_{0}}(\kappa) / s, s \rightarrow 0, \kappa \rightarrow 0 .
$$

Making use of the definition of the Riesz fractional derivative $R_{x}^{\alpha}$ as a pseudo differential operator with the symbol $-|\kappa|^{\alpha}$, i.e.,

$$
\mathcal{F}\left\{\left(R_{x}^{\alpha} f\right)(x) ; \kappa\right\}=-|\kappa|^{\alpha} \mathcal{F}\{f(x) ; \kappa\}
$$

and employing the Laplace transform formula

$$
\mathcal{L}\left\{\left(I_{t}^{\beta} f\right)(t) ; s\right\}=s^{-\beta} \tilde{f}(s), \beta>0
$$

for the Riemann-Liouville fractional integral defined by

$$
\left(I_{t}^{\beta} f\right)(t):=\left\{\begin{array}{l}
\frac{1}{\Gamma(\beta)} \int_{0}^{t}(t-\tau)^{\beta-1} f(\tau) d \tau, \beta>0, \\
f(t), \beta=0
\end{array}\right.
$$

the equation (2.14) can be rewritten in form of the fractional integro-differential equation

$$
\left.u(x, t)-u_{0}(x) \approx C_{\alpha}\left(I_{t}^{\frac{\alpha}{2}}\left(R_{x}^{\alpha} u\right)(x)\right)(t)\right)
$$

for large $t$ and $|x|$.

By applying the time-fractional differential operator $D_{t}^{\frac{\alpha}{2}}$ to the both sides of the equation (2.16), we can finally represent our model for large $t$ and $|x|$ in form of the initial-value problem

$$
u(x, 0)=u_{0}(x)
$$

for the time- and space-fractional diffusion equation ( $\alpha$-fractional diffusion equation)

$$
\left(D_{t}^{\frac{\alpha}{2}} u\right)(t) \approx C_{\alpha}\left(R_{x}^{\alpha} u\right)(x) .
$$


In what follows, the time-fractional derivative $D_{t}^{\beta}(m-1<\beta \leq m, m \in \mathbb{N})$ will be defined in the Caputo sense:

$$
\left(D_{t}^{\beta} f\right)(t)=\left(I_{t}^{m-\beta} f^{(m)}\right)(t) .
$$

It is worth mentioning that the integro-differential nature of the time-fractional differential operator in the model (2.17) ensures the non-Markovian nature of the diffusion process that is governed by this equation. Furthermore, both the mean waiting time and the jump length variance of the underlying continuous time random walk do not exist. Still, the model of the anomalous diffusion in form (2.17) possesses several interesting properties and is worth to be investigated.

\section{Analysis of the $\alpha$-fractional diffusion equation}

\subsection{Problem formulation}

Motivated by the continuous time random walk model introduced in the previous section, in this section we deal with the initial-value problem

$$
u(x, 0)=\varphi(x), \quad x \in \mathbb{R}^{2}
$$

for the two-dimensional $\alpha$-fractional diffusion equation

$$
D_{t}^{\frac{\alpha}{2}} u(x, t)=R_{x}^{\alpha} u(x, t), \quad x \in \mathbb{R}^{2}, \quad t \in \mathbb{R}_{+}, 0<\alpha \leq 2 .
$$

In (3.2), $u=u(x, t), x \in \mathbb{R}^{2}, t \in \mathbb{R}_{+}$is a real field variable, $R_{x}^{\alpha}$ is the Riesz space-fractional derivative of order $\alpha$ and $D_{t}^{\frac{\alpha}{2}}$ is the Caputo time-fractional derivative of order $\frac{\alpha}{2}$ defined by the formula (2.18). All quantities in (3.2) are assumed to be dimensionless, so that the coefficient by the Riesz space-fractional derivative can be taken to be equal to one without loss of generality.

As we already mentioned in the previous section, for a sufficiently well-behaved function $f: \mathbb{R}^{n} \rightarrow \mathbb{R}$, the Riesz space-fractional derivative of order $\alpha, 0<\alpha \leq 2$ is defined as a pseudo-differential operator with the symbol $-|\kappa|^{\alpha}$. For $0<\alpha<2, \alpha \neq 1$, it can be also represented as a hypersingular integral

$$
\left(R_{x}^{\alpha} f\right)(x)=-\frac{1}{d_{n, \ell}(\alpha)} \text { p.v. } \int_{\mathbb{R}^{n}} \frac{\left(\Delta_{h}^{\ell} f\right)(x)}{|h|^{n+\alpha}} d h
$$

with the finite differences operator $\left(\Delta_{h}^{\ell} f\right)(x)=\sum_{k=0}^{\ell}(-1)^{k}\left(\begin{array}{l}\ell \\ k\end{array}\right) f(x-k h)$ and a suitable normalization constant $d_{n, l}(\alpha)$ (see [31] for more details). In particular, in the one-dimensional case we get the representation

$$
\left(R_{x}^{\alpha} f\right)(x)=-\frac{1}{2 \Gamma(-\alpha) \cos (\alpha \pi)} \text { p.v. } \int_{0}^{\infty} \frac{f(x+\xi)-2 f(\xi)+f(x-\xi)}{\xi^{\alpha+1}} d \xi
$$

that is valid for $0<\alpha<2, \alpha \neq 1$.

For $\alpha=2$, equation (3.2) is reduced to the classical two-dimensional diffusion equation. In what follows, we focus on the case $0<\alpha<2$ because the case $\alpha=2$ (classical diffusion equation) is well studied in the literature.

\subsection{Fundamental solution of the $\alpha$-fractional diffusion equation}

The solution to the initial-value problem (3.1) for the $\alpha$-fractional diffusion equation (3.2) with the initial condition $\varphi(x)=\delta(x), x \in \mathbb{R}^{2}, \delta$ being the Dirac $\delta$-function is referred to as its fundamental solution and will be denoted by $G_{\alpha}=G_{\alpha}(x, t)$ in the further discussions. Surprisingly, the fundamental solution $G_{\alpha}$ can be derived in explicit form in terms of the Mittag-Leffler function. To get this representation, let us first apply the two-dimensional Fourier transform (2.9) to the $\alpha$-fractional diffusion equation (3.2) and to the initial conditions $(3.1)$ with $\varphi(x)=\delta(x)$. It follows from the definition of the Riesz space-fractional 
derivative as a pseudo-differential operator with the symbol $-|\kappa|^{\alpha}$ that the Fourier transform $\hat{G}_{\alpha}$ of the fundamental solution $G_{\alpha}$ satisfies both the initial condition

$$
\hat{G}_{\alpha}(\kappa, 0)=1
$$

and the ordinary fractional differential equation

$$
\left(D_{t}^{\frac{\alpha}{2}} \hat{G}_{\alpha}(\kappa, t)\right)(t)+|\kappa|^{\alpha} \hat{G}_{\alpha}(\kappa, t)=0 .
$$

The initial-value problem (3.4)-(3.5) possesses the unique solution (see e.g. [18])

$$
\hat{G}_{\alpha}(\kappa, t)=E_{\frac{\alpha}{2}}\left(-|\kappa|^{\alpha} t^{\frac{\alpha}{2}}\right)
$$

in terms of the Mittag-Leffler function

$$
E_{\beta}(z)=\sum_{k=0}^{\infty} \frac{z^{k}}{\Gamma(1+\beta k)}, \beta>0
$$

To recover the fundamental solution in the space domain we use the fact that the $n$-dimensional inverse Fourier transform of the radial functions (spherically symmetric functions) can be represented in the form (see e.g. [31])

$$
\phi(|x|)=\mathcal{F}^{-1}\{\phi(|\kappa|) ; x\}=\frac{|x|^{1-\frac{n}{2}}}{(2 \pi)^{\frac{n}{2}}} \int_{0}^{\infty} \phi(\tau) \tau^{\frac{n}{2}} J_{\frac{n}{2}-1}(\tau|x|) d \tau,
$$

where $J_{\nu}$ denotes the Bessel function with the index $\nu$. In particular, the two-dimensional inverse Fourier transform of a radial function $\phi$ can be written down as follows

$$
\phi(|x|)=\mathcal{F}^{-1}\{\phi(|\kappa|) ; x\}=\frac{1}{2 \pi} \int_{0}^{\infty} \phi(\tau) \tau J_{0}(\tau|x|) d \tau,
$$

where the Bessel function with the index zero is defined, say, by its series representation

$$
J_{0}(z)=\sum_{k=0}^{\infty} \frac{(-1)^{k}}{k ! k !}\left(\frac{z}{2}\right)^{2 k} .
$$

Of course, the formula (3.9) is valid only under the condition that the integral on its right-hand side converges absolutely or at least conditionally.

Because the Fourier transform of the fundamental solution $G_{\alpha}$ given by the formula (3.6) is evidently a radial function, we can employ the formula (3.9) and thus arrive at the representation

$$
G_{\alpha}(x, t)=\frac{1}{2 \pi} \int_{0}^{\infty} E_{\frac{\alpha}{2}}\left(-\tau^{\alpha} t^{\frac{\alpha}{2}}\right) \tau J_{0}(\tau|x|) d \tau
$$

that is valid under the condition that the integral on its right-hand side is a convergent one.

Let us investigate the convergence of this integral starting from the case $x=\mathbf{0}=(0,0)$. Then $|x|=0$, $J_{0}(0)=1$ and it easily follows from the well-known asymptotic formula

$$
E_{\beta}(-x)=-\sum_{k=1}^{m} \frac{(-x)^{-k}}{\Gamma(1-\beta k)}+O\left(|x|^{-1-m}\right), m \in \mathbb{N}, x \rightarrow+\infty, 0<\beta<2
$$

for the Mittag-Leffler function that the integral on the right-hand side of the formula (3.10) converges only in the case $\alpha \geq 2$. For $\alpha<2$, the fundamental solution $G_{\alpha}$ has thus a singularity at the point $x=\mathbf{0}=(0,0)$. As we shall see in the next section, for $\alpha>0$ this singularity is integrable and thus does not lead to any problems in our further discussions. 
In the case $x \neq \mathbf{0}=(0,0)$, the asymptotics (3.11) of the Mittag-Leffler function and the known asymptotics of the Bessel function

$$
\begin{gathered}
J_{\nu}(r)=O\left(r^{\nu}\right) \text { as } r \rightarrow 0, \\
J_{\nu}(r)=\sqrt{\frac{2}{\pi r}} \cos (r-\pi(1+2 \nu) / 4)+O\left(r^{-\frac{3}{2}}\right) \text { as } r \rightarrow+\infty
\end{gathered}
$$

are employed to derive the condition $\alpha>\frac{3}{2}$ for the absolute and the condition $\alpha>\frac{1}{2}$ for the conditional convergence of the integral at the right-hand side of (3.10). Thus the representation (3.10) of the fundamental solution $G_{\alpha}$ is valid only for $\alpha>\frac{1}{2}$ and we temporally assume this condition to be valid. Later on, other representations of the fundamental solution will be deduced that are valid for all values of $\alpha$ between zero and two.

In what follows, the technique of the Mellin integral transform and the Mellin-Barnes integrals is employed to derive other important representations of the fundamental solution $G_{\alpha}$. For the readers convenience the definition of the Mellin integral transform and some of its properties useful for the further discussions are given below. The Mellin integral transform of a function $f: \mathbb{R}_{+} \rightarrow \mathbb{R}$ is defined by

$$
\mathcal{M}\{f(\tau) ; s\}=f^{*}(s)=\int_{0}^{+\infty} f(\tau) \tau^{s-1} d \tau, \quad s \in \mathbb{C}, \gamma_{1}<\Re(s)<\gamma_{2}
$$

and the inverse Mellin transform by

$$
\mathcal{M}^{-1}\left\{f^{*}(s) ; \tau\right\}=f(\tau)=\frac{1}{2 \pi i} \int_{\gamma-i \infty}^{\gamma+i \infty} f^{*}(s) \tau^{-s} d s,
$$

where $\tau>0$ and $\gamma=\Re(s), \gamma_{1}<\gamma<\gamma_{2}$. Let us denote by $\stackrel{\mathcal{M}}{\leftrightarrow}$ the juxtaposition of a function $f(\tau)$ with its Mellin transform $f^{*}(s)$. In the further discussions the following transformation rules are used:

$$
\begin{aligned}
& f(a \tau) \stackrel{\leftrightarrow}{\leftrightarrow} a^{-s} f^{*}(s), \quad a>0, \\
& \tau^{a} f(\tau) \stackrel{\mathcal{M}}{\leftrightarrow} f^{*}(s+a), \\
& f\left(\tau^{p}\right) \stackrel{\mathcal{M}}{\leftrightarrow} \frac{1}{|p|} f^{*}(s / p), \quad p \neq 0 .
\end{aligned}
$$

The Mellin convolution of two functions, $f_{1}$ and $f_{2}$, is defined by

$$
f(y)=\int_{0}^{+\infty} f_{1}(\tau) f_{2}(y / \tau) \frac{d \tau}{\tau} .
$$

The main operational property of the Mellin convolution is given by the Mellin convolution theorem that is read as follows:

$$
f^{*}(s)=f_{1}^{*}(s) f_{2}^{*}(s)
$$

$f$ being the Mellin convolution of $f_{1}$ and $f_{2}$. Let us now derive an important representation of the fundamental solution $G_{\alpha}$ in terms of a Mellin-Barnes integral. For $|x| \neq 0$, the right-hand side of the formula (3.10) can be interpreted as the Mellin convolution of the functions

$$
f_{1}(\tau)=E_{\frac{\alpha}{2}}\left(-t^{\frac{\alpha}{2}} \tau^{\alpha}\right) \text { and } f_{2}(\tau)=\frac{1}{\alpha \pi|x|^{2} \tau^{2}} J_{0}\left(\frac{1}{\tau}\right)
$$

at the point $y=\frac{1}{|x|}$.

Using the known Mellin transforms of the Mittag-Leffler and the Bessel functions (see e.g. [22]) and the transformation rules listed above, we get the following Mellin transform pairs:

$$
E_{\frac{\alpha}{2}}\left(-t^{\frac{\alpha}{2}} \tau^{\alpha}\right) \stackrel{\leftrightarrow}{\leftrightarrow} \frac{t^{-\frac{s}{2}}}{\alpha} \frac{\Gamma\left(\frac{s}{\alpha}\right) \Gamma\left(1-\frac{s}{\alpha}\right)}{\Gamma\left(1-\frac{s}{2}\right)}, 0<\Re(s)<\alpha,
$$




$$
\tau^{-2} J_{0}\left(\frac{1}{\tau}\right) \stackrel{\mathcal{M}}{\leftrightarrow}\left(\frac{1}{2}\right)^{s-1} \frac{\Gamma\left(1-\frac{s}{2}\right)}{\Gamma\left(\frac{s}{2}\right)}, \frac{1}{2}<\Re(s)<2 .
$$

Now we apply the Mellin convolution theorem and the inverse Mellin transform and arrive at the representation $(|x| \neq 0)$

$$
G_{\alpha}(x, t)=\frac{1}{\alpha \pi|x|^{2}} \frac{1}{2 \pi i} \int_{\gamma-i \infty}^{\gamma+i \infty} \frac{\Gamma\left(\frac{s}{\alpha}\right) \Gamma\left(1-\frac{s}{\alpha}\right)}{\Gamma\left(\frac{s}{2}\right)}\left(\frac{2 \sqrt{t}}{|x|}\right)^{-s} d s, \quad \frac{1}{2}<\gamma<\alpha
$$

of the fundamental solution $G_{\alpha}$ in terms of the Mellin-Barnes integral (Fox H-function). For the details regarding evaluation of the improper integrals of the Mellin convolution type we refer the interested readers to [19] or [22].

It is worth mentioning that the simple form of the representation (3.14) is due to the fact that both the Mellin transform of $f_{1}(\tau)=E_{\frac{\alpha}{2}}\left(-t^{\frac{\alpha}{2}} \tau^{\alpha}\right)$ and the one of $f_{2}(\tau)=\frac{1}{\alpha \pi|x|^{2} \tau^{2}} J_{0}\left(\frac{1}{\tau}\right)$ contains a Gammafunction with the same parameter, namely $\Gamma\left(1-\frac{s}{2}\right)$, that could be canceled in their product. This feature is valid only in the two-dimensional case, whereas the Mellin-Barnes integrals of the type (3.14) are essentially more complicated both in the one- and in the three-dimensional cases (see the very recent paper [12] for the results in the one-dimensional case).

Employing the variables substitutions $s \rightarrow-s$, then $s \rightarrow s-2$, and then $s \rightarrow \frac{s}{\alpha}$ in the Mellin-Barnes integral (3.14) we derive three other useful representations $(|x| \neq 0)$ :

$$
\begin{gathered}
G_{\alpha}(x, t)=\frac{1}{\alpha \pi|x|^{2}} \frac{1}{2 \pi i} \int_{\gamma-i \infty}^{\gamma+i \infty} \frac{\Gamma\left(-\frac{s}{\alpha}\right) \Gamma\left(1+\frac{s}{\alpha}\right)}{\Gamma\left(-\frac{s}{2}\right)}\left(\frac{|x|}{2 \sqrt{t}}\right)^{-s} d s, \quad-\alpha<\gamma<-\frac{1}{2}, \\
G_{\alpha}(x, t)=\frac{1}{4 \alpha \pi t} \frac{1}{2 \pi i} \int_{\gamma-i \infty}^{\gamma+i \infty} \frac{\Gamma\left(\frac{2}{\alpha}-\frac{s}{\alpha}\right) \Gamma\left(1-\frac{2}{\alpha}+\frac{s}{\alpha}\right)}{\Gamma\left(1-\frac{s}{2}\right)}\left(\frac{|x|}{2 \sqrt{t}}\right)^{-s} d s, \quad 2-\alpha<\gamma<\frac{3}{2}, \\
G_{\alpha}(x, t)=\frac{1}{4 \pi t} \frac{1}{2 \pi i} \int_{\gamma-i \infty}^{\gamma+i \infty} \frac{\Gamma\left(\frac{2}{\alpha}-s\right) \Gamma\left(1-\frac{2}{\alpha}+s\right)}{\Gamma\left(1-\frac{\alpha}{2} s\right)}\left(\left(\frac{|x|}{2 \sqrt{t}}\right)^{\alpha}\right)^{-s} d s, \quad \frac{2}{\alpha}-1<\gamma<\frac{3}{2 \alpha} .
\end{gathered}
$$

The last representation can be used to derive a series representation of the fundamental solution. Indeed, taking into consideration the poles of the Gamma-function $\Gamma\left(1-\frac{2}{\alpha}+s\right)$ in the form $s_{k}=\frac{2}{\alpha}-1-k, k=$ $0,1,2 \ldots$ and applying the Jordan lemma and the Cauchy residue theorem, we get

$$
\begin{gathered}
G_{\alpha}(x, t)=\frac{1}{4 \pi t} \sum_{k=0}^{\infty} \frac{\Gamma(k+1)}{\Gamma\left(\frac{\alpha}{2}+\frac{\alpha}{2} k\right)} \frac{(-1)^{k}}{k !}\left(\left(\frac{|x|}{2 \sqrt{t}}\right)^{\alpha}\right)^{k+1-\frac{2}{\alpha}}= \\
\frac{1}{4 \pi t}\left(\frac{|x|}{2 \sqrt{t}}\right)^{\alpha-2} \sum_{k=0}^{\infty} \frac{1}{\Gamma\left(\frac{\alpha}{2}+\frac{\alpha}{2} k\right)}\left(-\left(\frac{|x|}{2 \sqrt{t}}\right)^{\alpha}\right)^{k} .
\end{gathered}
$$

The series in the last formula is nothing else than the Mittag-Leffler function with two parameters and we thus arrive at the following important and simple representation of the fundamental solution $G_{\alpha}$ in the form

$$
G_{\alpha}(x, t)=\frac{1}{4 \pi t}\left(\frac{|x|}{2 \sqrt{t}}\right)^{\alpha-2} E_{\frac{\alpha}{2}, \frac{\alpha}{2}}\left(-\left(\frac{|x|}{2 \sqrt{t}}\right)^{\alpha}\right),
$$

where the Mittag-Leffler function $E_{\beta, \gamma}$ is defined as follows:

$$
E_{\beta, \gamma}(z)=\sum_{k=0}^{\infty} \frac{z^{k}}{\Gamma(\beta k+\gamma)}, \beta>0
$$


Because $E_{1,1}(z)=\exp (z)$, in the case $\alpha=2$ the formula (3.18) reduces to the well-known formula

$$
G_{2}(x, t)=\frac{1}{4 \pi t} \exp \left(-\frac{|x|^{2}}{4 t}\right)
$$

for the fundamental solution to the classical two-dimensional diffusion equation.

For another "extremal" value of $\alpha$, namely, for $\alpha=1$ we can employ the formula (see e.g. [5])

$$
E_{\frac{1}{2}, \frac{1}{2}}(-z)=\frac{1}{\sqrt{z}}-z \exp \left(z^{2}\right)[1-\operatorname{erf}(z)]
$$

and experess the fundamental solution $G_{1}$ in the form:

$$
G_{1}(x, t)=\frac{1}{4 \pi t}\left(\frac{|x|}{2 \sqrt{t}}\right)^{-1}\left(\frac{1}{\sqrt{\frac{|x|}{2 \sqrt{t}}}}-\frac{|x|}{2 \sqrt{t}} \exp \left(\frac{|x|^{2}}{4 t}\right)\left[1-\operatorname{erf}\left(\frac{|x|}{2 \sqrt{t}}\right)\right]\right) .
$$

It is worth mentioning that in the case of a rational parameter $\alpha$ the Mittag-Leffler function $E_{\frac{\alpha}{2}}, \frac{\alpha}{2}$ can be represented as a linear combination of the generalized hypergeometric functions with the power weights and thus many other particular cases of the fundamental solution $G_{\alpha}$ can be deduced.

\section{Fundamental solution as a probability density function}

It is a well-known that the fundamental solution to the classical $n$-dimensional isotropic diffusion equation can be interpreted as a probability density function. In this section, we prove that the fundamental solution $G_{\alpha}$ to the two-dimensional $\alpha$-fractional diffusion equation is a spatial probability density function evolving in time, too.

Indeed, for $0<\alpha \leq 2$ the Mittag-Leffler function $E_{\frac{\alpha}{2}}, \frac{\alpha}{2}(-r), r \geq 0$ is a completely monotone function (see e.g. [5]) and thus nonnegative. It follows from the representation (3.18) that the fundamental solution $G_{\alpha}$ is nonnegative for $|x| \neq 0$ and $t>0$, too, i.e,

$$
G_{\alpha}(x, t) \geq 0,|x| \neq 0, t>0 .
$$

As to the point $x=(0,0)$, the fundamental solution tends to $+\infty$. But its asymptotic behavior is given by the expression (see the formula (3.18))

$$
G_{\alpha}(x, t)=O\left(|x|^{\alpha-2}\right) \text { as } x \rightarrow(0,0)
$$

and thus the fundamental solution is integrable at the point $x=(0,0)$.

Because of the well-known asymptotic formula

$$
E_{\beta, \gamma}(-x)=-\sum_{k=1}^{m} \frac{(-x)^{-k}}{\Gamma(\gamma-\beta k)}+O\left(|x|^{-1-m}\right), m \in \mathbb{N}, x \rightarrow+\infty, 0<\beta<2
$$

for the two-parametric Mittag-Leffler function, we first get the formula

$$
E_{\frac{\alpha}{2}, \frac{\alpha}{2}}\left(-\left(\frac{|x|}{2 \sqrt{t}}\right)^{\alpha}\right)=O\left(|x|^{-2 \alpha}\right),|x| \rightarrow+\infty
$$

and then the formula

$$
G_{\alpha}(x, t)=O\left(|x|^{-\alpha-2}\right) \text { as }|x| \rightarrow+\infty
$$

for asymptotics of the fundamental solution at infinity. Thus $G_{\alpha}$ is integrable at infinity, too. 
Let us now calculate the (convergent) improper integral

$$
I(t)=\int_{\mathbb{R}^{2}} G_{\alpha}(x, t) d x .
$$

To do this, one could use e.g. the technique of the Mellin transform and the Mellin convolution theorem. Another very efficient way to calculate this integral employs the relation

$$
I(t)=\left.\hat{G}_{\alpha}(\kappa, t)\right|_{\kappa=(0,0)}
$$

between this integral and the Fourier transform of the fundamental solution. The formula (3.6) for $\hat{G}_{\alpha}$ immediately leads to the final result:

$$
I(t)=\left.\hat{G}_{\alpha}(\kappa, t)\right|_{\kappa=(0,0)}=E_{\frac{\alpha}{2}}(0)=1 .
$$

The last property along with the non-negativity of $G_{\alpha}$ means that the fundamental solution to the $\alpha$ fractional diffusion equation can be interpreted as a two-dimensional probability density function evolving in time for all $\alpha, 0<\alpha \leq 2$. To the best knowledge of the author, the two-dimensional pdfs of the type (3.18) have been not yet introduced and investigated in the literature. Because in the framework of our model no correlation between the two directions on the plane was introduced, the two-dimensional pdf given by the formula (3.18) should be theoretically splittable into the product of two one-dimensional pdfs, but it is not straightforward to derive this splitting from the formula (3.18) and we let this analytical question for the future.

With the only exception for $\alpha=2$, the second moment of the pdf $G_{\alpha}, 0<\alpha \leq 2$ does not exist (see the asymptotic formula (4.3)) and thus the underlying stochastic model describes a kind of anomalous diffusion with the long-tailed waiting times and the long-tailed jump lengths. Because of the special asymptotics of the waiting time pdf and the jump length pdf (see the formulas (2.11) and (2.12)), the long waiting times are in a balance with the long jumps and thus this anomalous diffusion shows some characteristic features of the conventional diffusion, too. In particular, we show now that the entropy production rate of the anomalous diffusion described by the $\alpha$-fractional diffusion equation is the same as in the case of the conventional diffusion (of course, from the mathematical viewpoint this property is a consequence of the fact that the quotient of the orders of the time- and space-fractional derivatives in the $\alpha$-fractional diffusion equation is equal to one half like in the case of the conventional diffusion equation).

For a two-dimensional continuous random variable evolving in time with the pdf $p(x, t), x \in \mathbb{R}^{2}$ the Shannon entropy $S$ is defined by the formula

$$
S(p, t)=-\int_{\mathbb{R}^{2}} p(x, t) \ln (p(x, t)) d x
$$

and the entropy production rate is given by the relation

$$
R(p, t)=\frac{d}{d t} S(p, t)
$$

It is known that the entropy production rate is a very important characteristic of a stochastic process that can be interpreted as a natural measure of its irreversibility.

Say, the entropy of the two-dimensional Gaussian random variable evolving in time with the pdf (the fundamental solution to the classical two-dimensional diffusion equation)

$$
G_{2}(x, t)=\frac{1}{4 \pi t} \exp \left(-\frac{|x|^{2}}{4 t}\right)
$$

is given by the formula

$$
S\left(G_{2}, t\right)=1+\ln (4 \pi)+\ln (t)
$$


and thus its entropy production rate

$$
R\left(G_{2}, t\right)=\frac{d}{d t} S\left(G_{2}, t\right)=\frac{1}{t}
$$

is strictly positive (so that the corresponding diffusion process can be classified as an irreversible process) and decreases with the time.

It is worth mentioning that the entropy production rates for the time- and the space-fractional diffusion equations that were considered in [8] and [30], respectively, depend on the derivative order $\alpha$ and increase with increasing of $\alpha$ from one (diffusion) to two (wave propagation) that results in the so called entropy production paradox (see [8] and [30] for resolving of this paradox).

To calculate the entropy for the pdf defined by (3.18) (fundamental solution of the two-dimensional $\alpha$-fractional diffusion equation), we employ the relation (3.16) and represent it in the form $(t>0,|x| \neq 0)$

$$
G_{\alpha}(x, t)=\frac{1}{t} L_{\alpha}\left(\frac{|x|}{2 \sqrt{t}}\right), \quad L_{\alpha}(z)=\frac{1}{4 \alpha \pi} \frac{1}{2 \pi i} \int_{\gamma-i \infty}^{\gamma+i \infty} \frac{\Gamma\left(\frac{2}{\alpha}-\frac{s}{\alpha}\right) \Gamma\left(1-\frac{2}{\alpha}+\frac{s}{\alpha}\right)}{\Gamma\left(1-\frac{s}{2}\right)} z^{-s} d s
$$

in terms of an auxiliary function $L_{\alpha}$ that depends on the scaling variable $\frac{|x|}{2 \sqrt{t}}$. Of course, the representation (3.18) allows us to represent the function $L_{\alpha}$ in terms of the Mittag-Leffler function:

$$
L_{\alpha}(z)=\frac{1}{4 \pi} z^{\alpha-2} E_{\frac{\alpha}{2}, \frac{\alpha}{2}}(-z) .
$$

Let us also mention the Mellin transform formula for the function $L_{\alpha}$ that will be useful in the further discussions:

$$
L_{\alpha}^{*}(s)=\frac{1}{4 \alpha \pi} \frac{\Gamma\left(\frac{2}{\alpha}-\frac{s}{\alpha}\right) \Gamma\left(1-\frac{2}{\alpha}+\frac{s}{\alpha}\right)}{\Gamma\left(1-\frac{s}{2}\right)}=\frac{1}{4 \alpha \pi} \frac{\Gamma\left(\frac{s}{2}\right) \sin \left(\pi\left(1-\frac{s}{2}\right)\right)}{\sin \left(\pi\left(\frac{2}{\alpha}-\frac{s}{\alpha}\right)\right)} .
$$

This formula is obtained from the Mellin-Barnes representation (inverse Mellin transform formula) of $L_{\alpha}$ given in (4.5) and then using the Euler reflection formula for the Gamma-function.

With the representation (4.5) the formula for the entropy of the fundamental solution $G_{\alpha}$ takes the form

$$
S\left(G_{\alpha}, t\right)=-\int_{\mathbb{R}^{2}} \frac{1}{t} L_{\alpha}\left(\frac{|x|}{2 \sqrt{t}}\right) \ln \left(\frac{1}{t} L_{\alpha}\left(\frac{|x|}{2 \sqrt{t}}\right)\right) d x .
$$

Because the function in the integral on the right-hand side is a radial one, polar variables substitution leads to the expression

$$
S\left(G_{\alpha}, t\right)=-\int_{0}^{2 \pi} \int_{0}^{+\infty} \frac{1}{t} L_{\alpha}\left(\frac{r}{2 \sqrt{t}}\right) \ln \left(\frac{1}{t} L_{\alpha}\left(\frac{r}{2 \sqrt{t}}\right)\right) r d r d \phi
$$

and then to the one-dimensional integral in the form

$$
S\left(G_{\alpha}, t\right)=-\frac{2 \pi}{t} \int_{0}^{+\infty} L_{\alpha}\left(\frac{r}{2 \sqrt{t}}\right) \ln \left(\frac{1}{t} L_{\alpha}\left(\frac{r}{2 \sqrt{t}}\right)\right) r d r
$$

After some elementary transformations of this integral we obtain the following result:

$$
S\left(G_{\alpha}, t\right)=\left[8 \pi \int_{0}^{\infty} L_{\alpha}(\tau) \tau d \tau\right] \ln (t)-8 \pi \int_{0}^{\infty} L_{\alpha}(\tau) \ln \left(L_{\alpha}(\tau)\right) \tau d \tau=A_{\alpha} \ln (t)+B_{\alpha},
$$

where

$$
A_{\alpha}=8 \pi \int_{0}^{\infty} L_{\alpha}(\tau) \tau d \tau, \quad B_{\alpha}=-8 \pi \int_{0}^{\infty} L_{\alpha}(\tau) \ln \left(L_{\alpha}(\tau)\right) \tau d \tau
$$



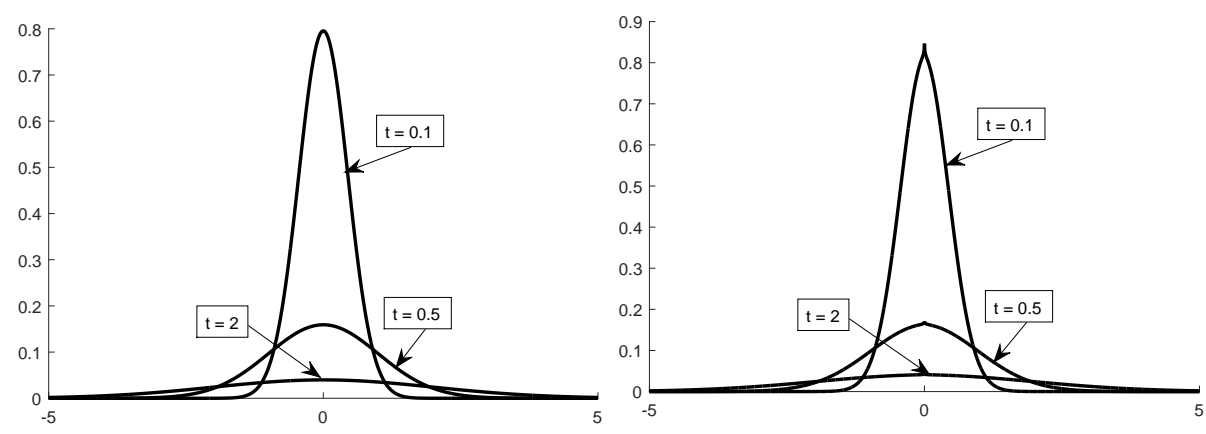

Figure 1. Plots of the fundamental solution $G_{\alpha}$ for $\alpha=2.0$ (left) and $\alpha=1.99$ (right)

Now we calculate the constant $A_{\alpha}$ in explicit form. To do this, let us note that the integral that defines $A_{\alpha}$ can be interpreted as the Mellin transform of $L_{\alpha}$ at the point $s=2$. The Mellin transform formula (4.6) leads then to the final result:

$$
A_{\alpha}=8 \pi \int_{0}^{\infty} L_{\alpha}(\tau) \tau d \tau=\frac{2}{\alpha} \lim _{s \rightarrow 2} \frac{\Gamma\left(\frac{s}{2}\right) \sin \left(\pi\left(1-\frac{s}{2}\right)\right)}{\sin \left(\pi\left(\frac{2}{\alpha}-\frac{s}{\alpha}\right)\right)}=\frac{2}{\alpha} \frac{\alpha}{2}=1 .
$$

This formula along with the formula (4.7) allows us to calculate the entropy production rate for the $\alpha$-fractional diffusion

$$
R\left(G_{\alpha}, t\right)=\frac{d}{d t} S\left(G_{\alpha}, t\right)=\frac{1}{t}
$$

that is exactly the same as the entropy production rate of a classical isotropic two-dimensional diffusion process.

\section{Plots and discussions}

In this section, some results of numerical evaluation of the fundamental solution $G_{\alpha}, 0<\alpha \leq 2$, its plots and discussions are presented.

The connection (3.18) between the fundamental solution $G_{\alpha}$ and the Mittag-Leffler function $E_{\frac{\alpha}{2}}$, $\frac{\alpha}{2}$ allows us to use well developed and reach theory of the Mittag-Leffler function (see e.g. the recent book [5] entirely devoted to the functions of the Mittag-Leffler type) for investigation of $G_{\alpha}$. In particular, the numerical algorithms for evaluation of the Mittag-Leffler function $E_{\beta, \gamma}$ suggested in [4] and the MATLAB-programs [34] that implement these algorithms can be used for numerical evaluation of the fundamental solution $G_{\alpha}$.

We start with the plots of the fundamental solution $G_{\alpha}$ with some fixed values of $\alpha$ at different time instants to demonstrate the time dynamics of the $\alpha$-diffusion for different values of $\alpha$. Because the fundamental solution $G_{\alpha}$ is a radial function, we restrict ourselves to producing just two-dimensional intersections of the three-dimensional surfaces $z=z\left(x_{1}, x_{2}\right)=G_{\alpha}(|x|, t)$ with the plane $x_{2}=0$. As can be seen on the plots of Figure 1, for values of $\alpha$ close to 2, say $\alpha=1.99$, the form of the fundamental solution $G_{\alpha}$ for the "middle" values of $|x|$ is very similar to one of the Gaussian distribution. Still, there are two very essential differences between $G_{2}$ and $G_{\alpha}$ even if $\alpha$ is very close to 2. On the one hand, $G_{\alpha}(|x|, t) \rightarrow+\infty$ as $|x| \rightarrow 0$ for $\alpha<2$. On the other hand, $G_{\alpha}$ with $\alpha<2$ has a power-law asymptotics as $|x| \rightarrow \infty$ in contrast to the exponential decay of $G_{2}$. The plots of Figure 2 demonstrate the form of the fundamental solution $G_{\alpha}$ for some values of $\alpha$ between one and two. We can see that the anomalous diffusion process that is governed by the corresponding $\alpha$-fractional diffusion equation becomes slower compared to the conventional diffusion, i.e., the probability of finding a diffusing particle near its initial position at a certain fixed time instant increases when $\alpha$ becomes smaller. On the other hand, because 

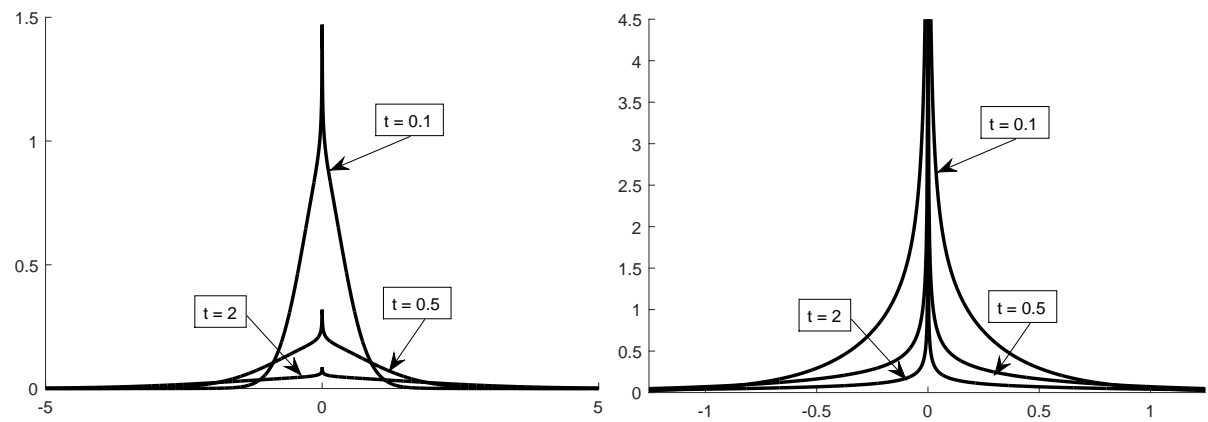

Figure 2. Plots of the fundamental solution $G_{\alpha}$ for $\alpha=1.9$ (left) and $\alpha=1.5$ (right)
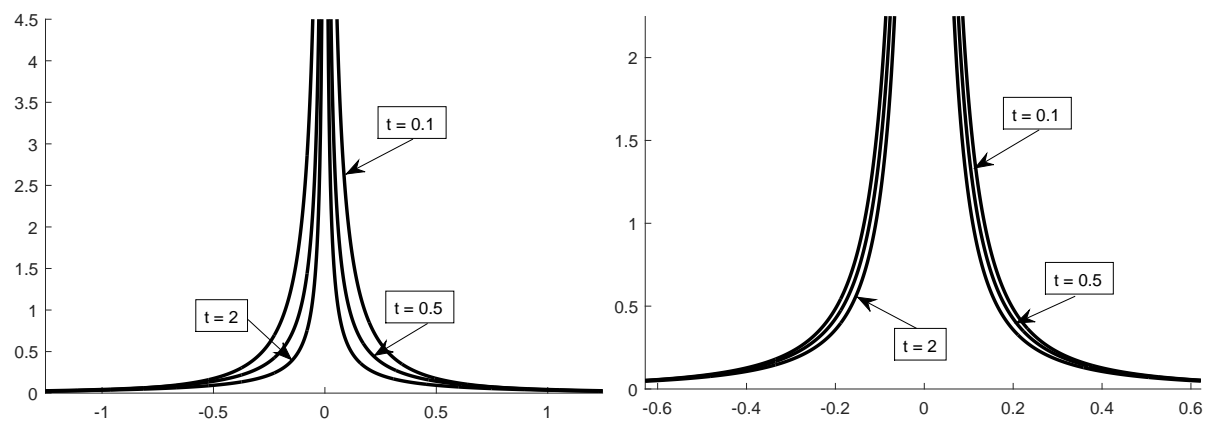

FiguRE 3. Plots of the fundamental solution $G_{\alpha}$ for $\alpha=1.0$ (left) and $\alpha=0.5$ (right)
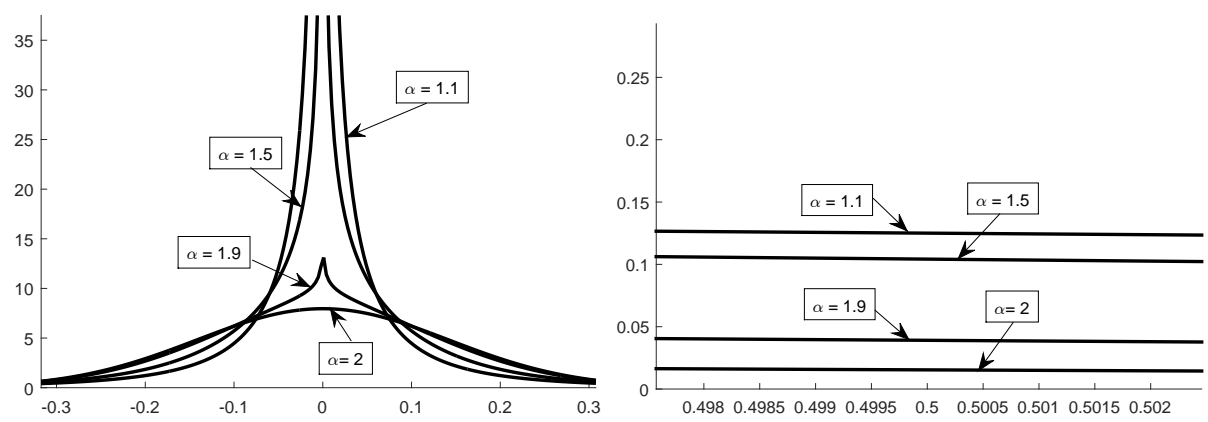

Figure 4. Plots of the fundamental solution $G_{\alpha}$ for $t=0.01$

of the long-tailed jump pdf of the underlying continuous time random walk model, the probability of positions located far away from the initial position increases when $\alpha$ becomes smaller, too. Finally, as we see on the plots of Figure 3, for $\alpha \leq 1$ the $\alpha$-fractional diffusion becomes extremely slow near the origin (see e.g. the second plot of Figure 3), but it still has a power-law asymptotics as $|x| \rightarrow \infty$ because of the long-tailed jump pdf of the underlying continuous time random walk model.

On the plots of Figures 4-7 a comparison between the plots of the fundamental solution $G_{\alpha}$ with different $\alpha$-values plotted at the same time instant is presented. At each time instant, the plots have three different regions that characterize the anomalous diffusion processes governed by the $\alpha$-fractional diffusion equation compared to the conventional diffusion. As can be seen on the left plots of Figures 

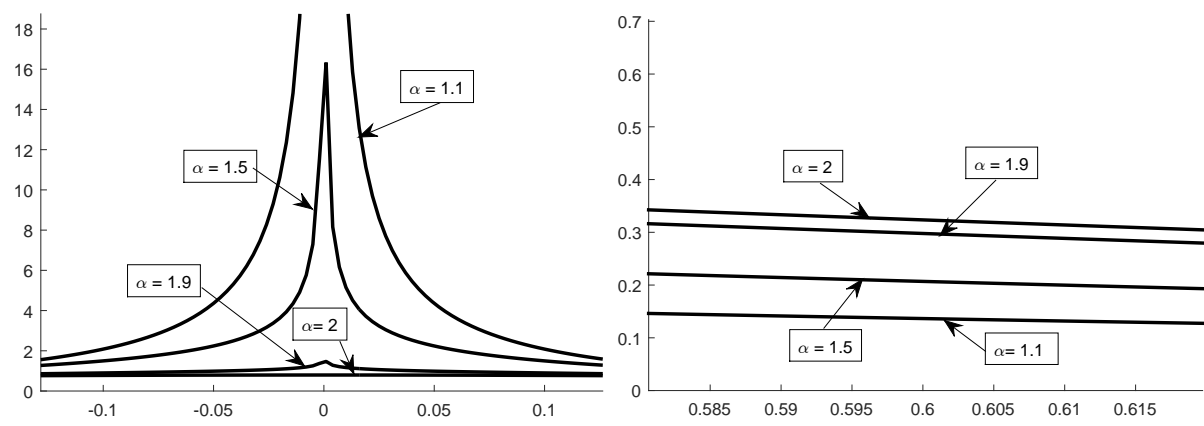

Figure 5. Plots of the fundamental solution $G_{\alpha}$ for $t=0.1$ for small (left) and "middle" (right) values of $|x|$
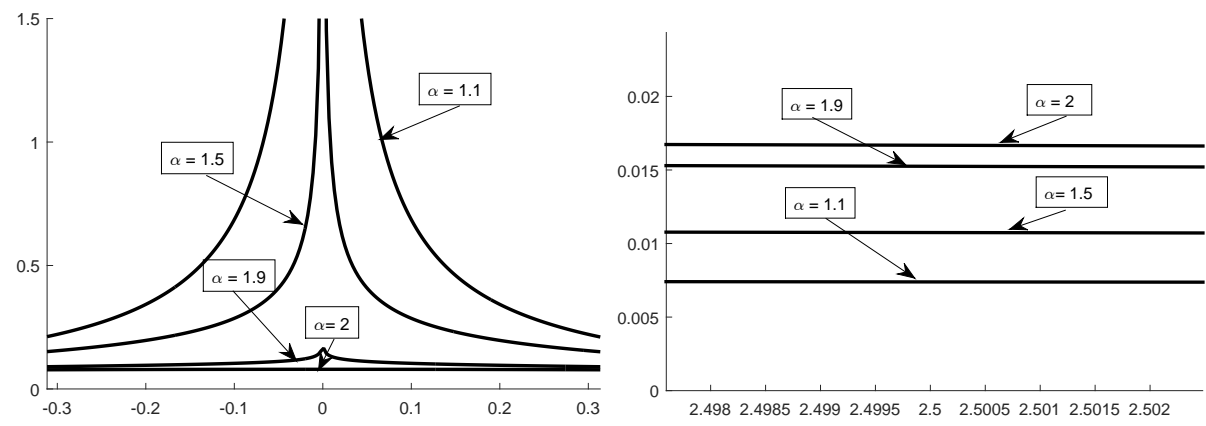

Figure 6 . Plots of the fundamental solution $G_{\alpha}$ for $t=1.0$ for small (left) and "middle" (right) values of $|x|$

4-6, the fundamental solution $G_{\alpha}$ increases near the origin with $\alpha$ decreasing from two to zero as a consequence of the long-tailed waiting time pdfs of the underlying continuous time random walk model. Because of the long-tailed jump length pdfs, $G_{\alpha}$ has a power-law asymptotic decay of the type $O\left(|x|^{-\alpha-2}\right)$ as $|x| \rightarrow \infty$ and thus the probability of locations located far away from the initial position increases when $\alpha$ becomes smaller, too, as can be seen on the right plot of Figure 4 and the plots of Figure 7. Finally, for the "middle" values of $|x|, G_{\alpha}$ increases with $\alpha$ increasing from zero to two (see the left plot of Figure 4 and the right plots of Figures 5 and 6).

Summarizing the discussions above, the $\alpha$-fractional diffusion equations with $0<\alpha<2$ could be useful for description of the anomalous diffusion processes that are characterized by the long-tailed waiting time pdfs (and thus larger concentration of the diffusing substance near the initial location compared to the conventional diffusion) and simultaneously by the long-tailed jump length pdfs (and thus larger concentration of the diffusing substance far away from the initial location compared to the conventional diffusion). Roughly speaking, the diffusing substance should mainly consist of both "fast" and "slow" diffusing particles compared to the "normal" particles in the framework of the conventional diffusion processes.

Acknowledgements. The author appreciates the constructive remarks and suggestions of the anonymous referees that helped to improve the manuscript. 

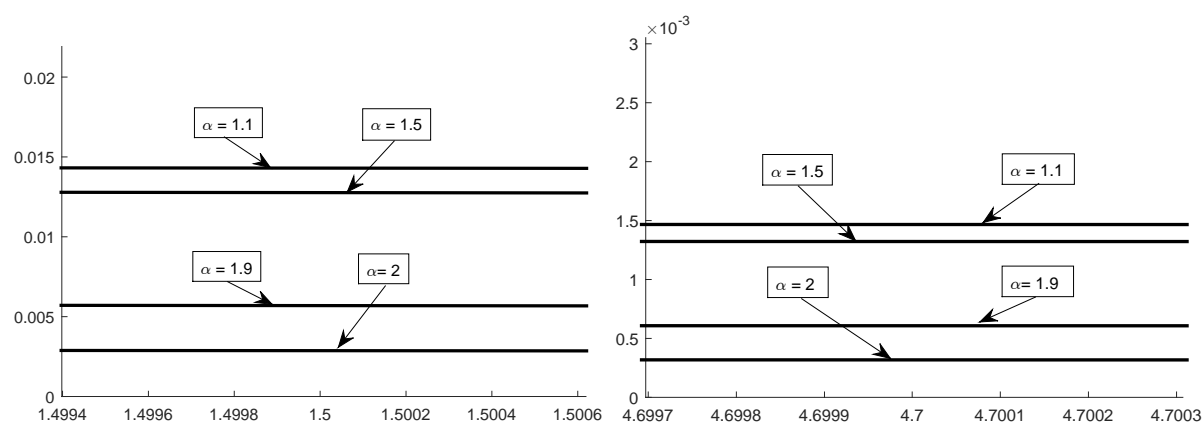

Figure 7. Plots of the fundamental solution $G_{\alpha}$ for $t=0.1$ (left) and $t=1.0$ (right) for "large" values of $|x|$

\section{References}

[1] G. Germano, M. Politi, E. Scalas, R.L. Schilling. Stochastic calculus for uncoupled continuous-time random walks. Physical Review E, 79 (2009), 066102.

[2] R. Gorenflo. Stochastic processes related to time-fractional diffusion-wave equation. Communications in Applied and Industrial Mathematics, 6 (2014), e-531.

[3] R. Gorenflo, F. Mainardi. Subordination Pathways to Fractional Diffusion. Eur. Phys. J. Special Topics, 193 (2011), 119-132.

[4] R. Gorenflo, J. Loutchko, Yu. Luchko. Computation of the Mittag-Leffler function and its derivatives. Fract. Calc. Appl. Anal., 5 (2002), 491-518.

[5] R. Gorenflo, A.A. Kilbas, F. Mainardi, S.V. Rogosin, Mittag-Leffler Functions, Related Topics and Applications. Springer, Berlin, 2014.

[6] A. Hanyga. Multi-dimensional solutions of space-time-fractional diffusion equations. Proc. R. Soc. London. A, 458 (2002), 429-450.

[7] R. Hilfer, L. Anton. Fractional master equations and fractal time random walks. Phys. Rev. E, Rapid Commun., 51 (1995), R848.

[8] K.H. Hoffmann, C. Essex, C. Schulzky. Fractional diffusion and entropy production. J. Non-Equilib. Thermodyn., 23 (1998), 166-175.

[9] M. Kenkre, E.W. Montroll, M.F. Shlesinger. Generalized master equations for continuous-time random walks. Journal of Statistical Physics, 9 (1973), 45-50.

[10] R. Klages, G. Radons, I.M. Sokolov. Anomalous Transport: Foundations and Applications. Wiley-VCH, 2008.

[11] X. Li, C. Essex, M. Davison, K.H. Hoffmann, C. Schulzky. Fractional diffusion, irreversibility and entropy. J. NonEquilib. Thermodyn., 28 (2003), 279-291.

[12] Yu. Luchko. Entropy production rate of a one-dimensional alpha-fractional diffusion process. Axioms, 5 (2016), doi:10.3390/axioms5010006.

[13] Yu. Luchko. Wave-diffusion dualism of the neutral-fractional processes. Journal of Computational Physics, 293 (2015), 40-52.

[14] Yu. Luchko. Multi-dimensional fractional wave equation and some properties of its fundamental solution. Communications in Applied and Industrial Mathematics, 6 (2014), e-485.

[15] Yu. Luchko. Fractional wave equation and damped waves. J. Math. Phys., 54 (2013), 031505.

[16] Yu. Luchko. Models of the neutral-fractional anomalous diffusion and their analysis. AIP Conf. Proc., 1493 (2012), 626-632.

[17] Yu. Luchko. Anomalous diffusion models and their analysis. Forum der Berliner mathematischen Gesellschaft, 19 (2011), 53-85.

[18] Yu. Luchko, R. Gorenflo. An operational method for solving fractional differential equations with the Caputo derivatives. Acta Mathematica Vietnamica, 24 (1999), 207-233.

[19] Yu. Luchko, V. Kiryakova. The Mellin integral transform in fractional calculus. Fract. Calc. Appl. Anal., 16 (2013), 405-430.

[20] M. Magdziarz, A. Weron, J. Klafter. Equivalence of the Fractional Fokker-Planck and Subordinated Langevin Equations: The Case of a Time-Dependent Force. Phys. Rev. Lett., 101 (2008), 210601.

[21] F. Mainardi, Yu. Luchko, G. Pagnini. The fundamental solution of the space-time fractional diffusion equation. Fract. Calc. Appl. Anal., 4 (2001), 153-192.

[22] O.I. Marichev. Handbook of Integral Transforms of Higher Transcendental Functions. Theory and Algorithmic Tables. Ellis Horwood, 1983. 
[23] R. Metzler, J.-H. Jeon, A.G. Cherstvy, E. Barkai. Anomalous diffusion models and their properties: non-stationarity, non-ergodicity, and ageing at the centenary of single particle tracking. Phys. Chem. Chem. Phys., 16 (2014), 2412824164

[24] R. Metzler, J. Klafter. The restaurant at the end of the random walk: Recent developments in the description of anomalous transport by fractional dynamics. J. Phys. A. Math. Gen., 37 (2004), R161-R208.

[25] R. Metzler, J. Klafter. The random walk's guide to anomalous diffusion: a fractional dynamics approach. Physics Reports, 339 (2000), 1-77.

[26] A. Mura, G. Pagnini. Characterizations and simulations of a class of stochastic processes to model anomalous diffusion. J. Phys. A: Math. Theor., 41 (2008), 285003.

[27] G. Pagnini. Short note on the emergence of fractional kinetics. Physica A, 409 (2014), $29-34$.

[28] G. Pagnini. Erdélyi-Kober fractional diffusion. Fract. Calc. Appl. Anal., 15 (2012), 117-127.

[29] Yu. Povstenko. Linear Fractional Diffusion-Wave Equation for Scientists and Engineers. Birkhäuser, 2015.

[30] J. Prehl, C. Essex, K.H. Hoffmann. The superdiffusion entropy production paradox in the space-fractional case for extended entropies. Physica A, 389 (2010), 214-224.

[31] S.G. Samko, A.A. Kilbas, O.I. Marichev. Fractional Integrals and Derivatives: Theory and Applications. Gordon and Breach, 1993.

[32] W.R. Schneider, W. Wyss. Fractional diffusion and wave equations. J. Math. Phys., 30 (1989), $134-144$.

[33] S. Umarov. Continuous time random walk models associated with distributed order diffusion equations. Fract. Calc. Appl. Anal., 18 (2015), 821-837.

[34] Matlab File Exchange, Matlab-Code that calculates the Mittag-Leffler function with desired accuracy, available for download at www.mathworks.com/matlabcentral/fileexchange/8738-mittag-leffler-function. 